\title{
A CASE STUDY OF AN ULTRASOUND CLINIC TO OPTIMIZE WAITING TIME USING QUEUING THEORY
}

\author{
PREETI DESHWAL ${ }^{1} \&$ NEETU GUPTA ${ }^{2}$ \\ ${ }^{I}$ Scholar in the Department of Mathematics, J.C.Bose University of Science and Technology, Y.M.C.A. Faridabad, India \\ ${ }^{2}$ Associate Professor in Mathematics, J.C.Bose University of Science and Technology, Y.M.C.A Faridabad, India
}

\begin{abstract}
With the changes in life styles and eatables of human beings we are facing so many health related problems in our daily lives. Many of them require CT scan, X-Ray, MRI, tomography, Ultrasound etc. Generally patients are dissatisfied with waiting. Minimum waiting time and good quality service are the two main expectations of patients from the clinic. Minimum waiting requires a good patient flow through various stages and it can be achieved by applying an appropriate queuing model. This paper aims to study the data set of an ultrasound lab to minimize the problem of waiting. Hence queuing theory can help in managing resource allocation of an ultrasound lab. So our main motive is to predict the patient's arrival pattern and using that information to plan tasks so that waiting can be reduced as much as possible.

KEYWORDS: Queuing Model, Healthcare \& Ultrasound Technology
\end{abstract}

Received: Jun 08, 2020; Accepted: Jun 28, 2020; Published: Aug 21, 2020; Paper Id.: IJMPERDJUN2020848

\section{INTRODUCTION}

Queuing theory is a branch of mathematics that studies and models the act of waiting in lines. Waiting lines or queues can be seen in any system where arrival rate is more than the system can handle. The first paper on queuing theory, "The Theory of Probabilities and Telephone Conversations" was published in 1909 by A.K. Erlang. No doubt that queuing theory was first applied to analyze the problem of telephone waiting calls by A.K. Erlang in 1913 but nowadays everybody is becoming aware of the applications of queuing theory in other areas. In developing a solution to this problem he began to realize that the problem of minimizing waiting time was applicable to many fields, and began developing the theory further. Queuing models are used for estimating waiting time of a patient, utilization of service, models system design, and models for evaluating appointment systems. A queuing system helps minimizing the waiting time of patients and maximizing the utilization of the servers i.e. doctors, nurses, hospital beds etc. Queuing is not new but recently hospitals have begun to use it effectively. After knowing the use of queuing models in determining the optimal allocation of resources in a hospital, hospital management teams are using it more and more. The need of applying queuing theory and queuing models in healthcare settings is because it can help to save someone's life.

Although there are different levels of healthcare centers but they all act in a similar fashion in terms of treating a patient. They all have a set of activities that every patient needs to undergo during the treatment.Moreover the rate of patient's admitting in public hospitals is more as compared to private hospitals because service provided in public hospitals is cheaper than that of private hospitals. With increasing population, admission in Emergency Departments is also increasing but due to limited amount of resources and facilities long waiting lines are seen. The goal is toprovide some solution to these waiting lines, delays. Therefore optimizing the use of Resources and 
providing better service is main motive of this study.

Managing patients waiting time, providing better service, optimizing the allocation of resources available is a big challenge for public hospitals all over the world. Long queues in the emergency departments, ultrasound clinics, Dental clinics may have negative impact on the patients. These queues are formed because of random arrivals of patients in hospitals. Because of complex and variable nature of patient's arrival it is very difficult to solve the problem without using new technologies. Using queuing theory and queuing models, it is possible to calculate the exact amount of resources required in a hospital. Ultrasoundtechnology is useful and has grownup very vastly in this developing world. It is beneficial for diagnosis of a disease at an early stage and getting treatment accordingly. It impacts patient's management plans positively.

\section{LITERATURE REVIEW}

Shah [16] has studied the impact of ultrasound services in rural Rwanda. They collected datasheets of ultrasounds performed and concluded that this technology is appearing most beneficial for pregnant and adult women. Moreover they also concluded that ultrasound service changed patient's management plans in $43 \%$ cases of total patients scanned. Doehring Schwerdtfeger [18] et al have studied the impact of ultrasound services and concluded that it was useful as diagnostic aid in obstetrics, pediatrics.

Samuel Fomundam and Jeffrey Herrmann[6] have used applications of queuing theory to study system design, appointment system, calculating waiting time and utilization analysis. S. Creemers et al[4] have used the concept of queuing theory to describe the impact of sudden and unplanned interrupts and absences of patients in a waiting list. Thandaaunget al[19] have done a comparative study on different queuing models. They analyzed the datasets of Brahmaso clinic in various departments such as registration department, pre-checkup department, consultation department, treatment and pharmacy department, emergency department etc. They have used multi-server queuing model to reduce waiting time and increase the efficiency of the clinic. They finally concluded that increasing number of servers is a good way of minimizing the waiting time of patients significantly.

Hadimohammad et al[3] have used queuing models to a local health region with a network of six services-acute care, long term care, assisted living, homecare, rehabilitation and chronic care.Lakshmi $\mathrm{C}$ et al[9] have contributed their research in the applications of queuing theory in healthcare. In their work they examined digital libraries, journals, books, conference papers. They concluded that most of the articles are published in or after 2000 and all this is because of advancement in technology. FatmaPoniMardiah et al[10] have analyzed data to study appointment system in a public hospital of Indonesia. Moreover their contribution is in reducing outpatient's waiting time in a hospital. In their study, they have concluded that hospital management team should focus on the four main quality indicating parameters of a healthcare system naming appointment system, waiting time and patient flow and capacity. Also they have explained the reasons why people prefer to go in a private hospital rather than government hospitals.

MohammadkarimBahadori et al[3] have analyzed a hospital pharmacy performance using queuing theory and simulation model. This study was conducted in military hospital in Iran; Tehran shows that queuing theory can act as a powerful tool to manage a pharmacy. They collected data regarding the time of filling prescription drugs for patients coming in morning and evening shifts using the preplanned forms. Collected data was further analyzed by queuing models. Moreover they analyzed the data collected for both morning and evening shifts and concluded that patient's waiting time 
can be reduced by assigning job to multitasking persons.Olorunsola S. A et al [21] considered the queuing analysis of patient flow in a hospital. Patient flow refers to progress of a patient's health status. They calculated various parameters like bed occupancy rate, length of stay distribution using M/M/C model. Finding this optimal number of beds in a hospital is a very common practice to enhance the service quality of a hospital. Using these parameters hospital managers can improve the service quality. They also explained how optimal number of beds effect patient flow in a hospital.

Hajnal Vass et al [22] have studied the concept of patient flow in an Emergency Department. This study was conducted in Romania. They have used M/M/c model to study the quality indicating parameters of the Emergency Department situated in Romania and reveals that that how queuing theory can help in optimal allocation of resources in an Emergency Department. Moreover they also analyzed that increasing the quality of service provided by the hospital will increase the cost but it is important to optimize the whole process.

McClain[11] reviews research on applying queuing models to study the impact of bed assignment polices on utilization, waiting time and the probability of turning away patients. Nkeiruka Amah et al [1] have done a survey to find patient's satisfaction rate in Nigerian hospital. They also find out the various causes of long waiting lines in hospitals. They calculated patient's satisfaction rate by having a questionnaire. Although queuing theory have a lots of applications but it has been used widely in service in service industries (Nosek and Wilson)[12]. They have used queuing theory in pharmacy area with special focus to improve customer's satisfaction.

\section{Working Process of Ultrasound Clinic}

Patients arrive in the ultrasound clinic through the main door and then come to reception. At the reception, patients are asked to get registered with their name and pay the fees. After that they are provided a unique number and they wait until that number is called for further process. When a patient's turn comes, they proceed with document verification, signature and photo of the party along with patient. After doing all formalities patient wait for getting ultrasound service. Finally they get the service and do a small consultation with the doctor and finally wait for collecting their reports. Lastly patients collect reports and depart from the ultrasound clinic.It was noticed that there is only one service station (reception) where patients arrive and each patient have to undergo through various stages of treatment process. The queue starts at registration desk and continue. Although there is awaiting room for patients but if it gets full the queue continue out of the waiting room indicating that the system capacity is infinite. Hence it in concluded that $\left\{\left(\mathrm{M} / \mathrm{E}_{\mathrm{k}} / 1\right)\right.$ : $\left.(\mathrm{FCFS} / \infty / \infty)\right\}$ queuing model best fits into the current situation. 
Arrival of patients in the clinic

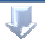

Registration at reception

$\sqrt{2}$

A nurse varifies essential documents for furthur treatment

业

Processing photos and signatures of patient and accompanied him

F

Ultrasound process

consultation and Collecting report from reception

$\sqrt{2}$

Exit from the clinic.

Figure 1: A Model of Patient Flow Through Ultrasound Clinic.

\section{Model Description}

The study adopted single server multi-phase service queuing model.

$\left\{\left(\mathrm{M} / \mathrm{E}_{\mathrm{k}} / 1\right):(\mathrm{FCFS} / \infty / \infty)\right\}$ Erlang service time distribution with $\mathrm{k}$ phases

M=Markovian (or Poisson) arrivals;

$\mathrm{E}_{\mathrm{k}}=$ Erlangian service time distribution;

FCFS $=$ First come, first served;

$\infty=$ Infinite system limit;

$\infty=$ Infinite source limit.

Patients have to join queue at each phase of the system. This model consists of single service channel in which there are $k$ identical stages(phases) in series,each with average service time $1 / k \mu$ as shown in figure1.The distribution of total service time of a customer in the system will be some combined distribution of time in all these phases.

Each customer is served in $k$-phases one by one and a new service does not start until all k-phases have been completed, therefore each arrival will increase the number of phases by $k$ in the system. Thus,if there are $m$ customers in the queue and one customer is already in service at the sth phase, then the total number of phasesin the system(waiting and in service) will be $n=m k+s$.

A multi phase single server Erlang queuing model is shown in the following diagram.

Customers in Queue Queuing System 


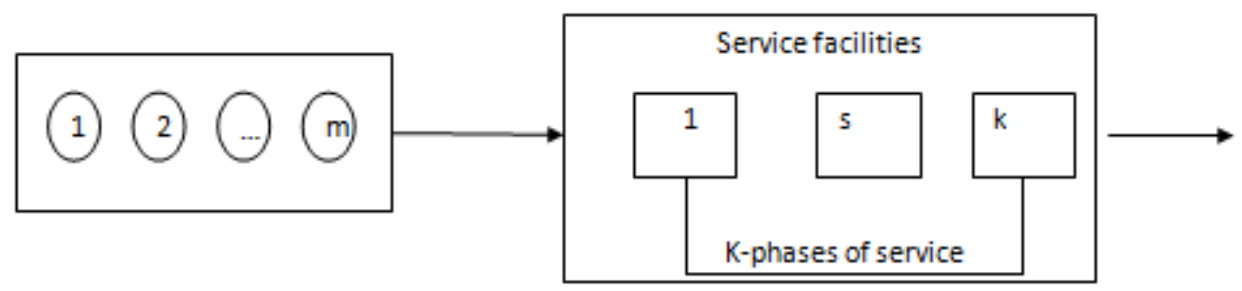

Figure 2: A Multi Phase Single Server Erlang Queuing Model

If $\mu$ denotes the number of customers per unit of time, then $k \mu$ will be the number of phases served per unit of time, and $1 / k \mu$ will be the average time taken by server at each phase.

Therefore

$\lambda_{\mathrm{n}}=\lambda$ phases arrive per unit time.

$\mu_{\mathrm{n}}=k \mu$ phases served per unit time.

The probability density function for Erlang distribution is:

$$
f(t)=\frac{(k \mu)^{k}}{(k-1) !} t^{k-1} e^{-k \mu t} ; t \geq 0
$$

Where $\mu=$ expected number of customers completing service per unit time

$k=\mathrm{a}$ positive integer

The expected total service time and variance of Erlang distribution is given by:

$$
E(t)=k\left(\frac{1}{k \mu}\right)=\frac{1}{\mu} \text { and } \sigma^{2}=k\left(\frac{1}{k \mu}\right)^{2}=\frac{1}{k \mu^{2}}
$$

For general value of $k$, the model value of service time, $\mathrm{t}$ is $(k-1) / k \mu$.

Figure 3 shows how the shape of Erlang distribution changes for various values of $k$, when $k=1$, it reduces to the exponential distribution, whereas for $1<k<\infty$, it reduces to a constant distribution for customer inter arrival times.

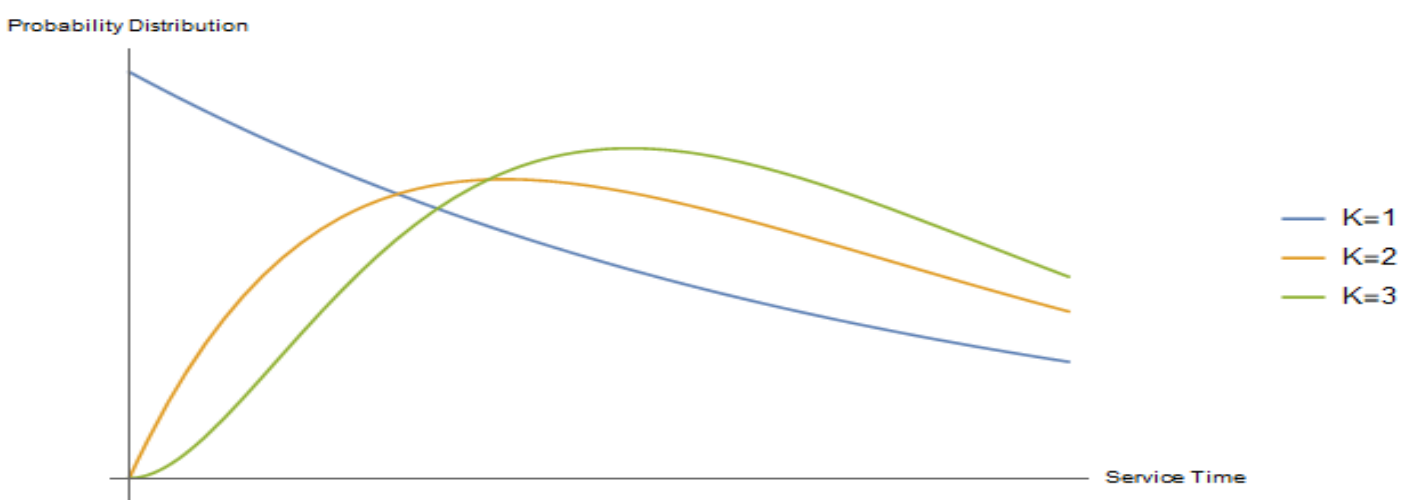

Figure 3: The Shape of Erlang Distribution for Various Values of $k$. 


\section{Calculations}

The ultrasound clinic works from 9a.m to 6p.m. On seeing the arrival pattern of patients we partition the data into seven different days of the week. Total patients coming to a particular day divided by nine (number of working hours of the clinic) gives us average arrival rate for that particular day. Discussion with the hospital manager helps us to find that there are three stages in the whole process of ultrasound service. At first the patient arrives and goes to reception for registration. First stage consists of verification of necessary documents by a hospital nurse and a computer operator takes photo of the patient and accompanied person using a web camera. In second phase the patient goes to doctor's cabin for ultrasound. Third stage consists of consultation process and patients depart from the clinic after collecting reports from the reception. It was calculated that on an average 2.3minutes are taken to complete each phase of the service process. Therefore mean service time of a patient comes 6.9minutesapproximately. Moreover we also noticed that service rate remains the same during all weekdays although the arrival rate varies. The following table shows the arrival rate and departure rate of patients in different days of the week.

Table 1: Input Parameters of $\{(\mathrm{M} / \mathrm{Ek} / \mathbf{1})$ : (FCFS/ $/ \infty)\}$ Model.

\begin{tabular}{|l|c|c|}
\hline Day of the week & Arrival Rate $(\boldsymbol{\lambda})$ per Hour & Service Rate $(\mathbf{k} \boldsymbol{\mu})$ Per Hour \\
\hline Sunday & 8.6 & 8.69 \\
\hline Monday & 8.16 & 8.69 \\
\hline Tuesday & 6.16 & 8.69 \\
\hline Wednesday & 6.69 & 8.69 \\
\hline Thursday & 7.42 & 8.69 \\
\hline Friday & 7.08 & 8.69 \\
\hline Saturday & 7.62 & 8.69 \\
\hline
\end{tabular}

- The expected numbers of phases(not customers) in the queue,

$$
L_{q}(k)=\frac{L_{s}(k)}{\mu}=\frac{1+k}{2} \frac{\lambda}{\mu(\mu-\lambda)}
$$

- 2. The expected numbers of customers (not phases) in the queue,

$L q=L_{s}(k)$-average number of patients in service

$$
\frac{1}{k}\left[\frac{k+1}{2} \frac{\lambda}{\mu-\lambda}-\frac{k+1}{2} \frac{\lambda}{\mu}\right]=\frac{1+k}{2 k} \frac{\lambda^{2}}{\mu(\mu-\lambda)}
$$

- 3. The expected waiting time before being served, $W_{q}=\frac{1+k}{2 k} \frac{\lambda}{\mu(\mu-\lambda)}$

- 4. The expected time spent in the system, $W_{s}=W_{q}+\frac{1}{\mu}$

- 5. The expected numbers of customers in the system, $L_{s}=\lambda W_{s}$

Using the formulas and input parameters from the table1 the following table shows the calculated values of various output parameters. 
Table 2: The Calculated Values of Various Output Parameters.

\begin{tabular}{|l|c|c|c|c|c|}
\hline \multicolumn{1}{|c|}{ Day of the Week } & $\boldsymbol{L q}(\boldsymbol{k})$ & $\boldsymbol{L q}$ & $\boldsymbol{W}_{\boldsymbol{q}}$ & $\boldsymbol{W}_{\boldsymbol{s}}$ & $\boldsymbol{L}_{\boldsymbol{s}}$ \\
\hline Sunday & 21.99 & 63.04 & 7.33 & 7.45 & 64.03 \\
\hline Monday & 3.54 & 9.64 & 1.18 & 1.30 & 10.58 \\
\hline Tuesday & 0.56 & 1.15 & 0.19 & 0.30 & 1.86 \\
\hline Wednesday & 0.77 & 1.72 & 0.26 & 0.37 & 2.49 \\
\hline Thursday & 1.34 & 3.33 & 0.45 & 0.56 & 4.18 \\
\hline Friday & 1.01 & 2.39 & 0.34 & 0.45 & 3.20 \\
\hline Saturday & 1.64 & 4.16 & 0.55 & 0.66 & 5.04 \\
\hline
\end{tabular}

\section{Data Presentation}

In a period of one month total 2015 patients registered themselves to get ultrasound service. These patients have different health issues such as kidney stone, ovarian cyst, Tumor TB, HIV, AIDS etc. The following figure represents percentage of total patients having different problems.

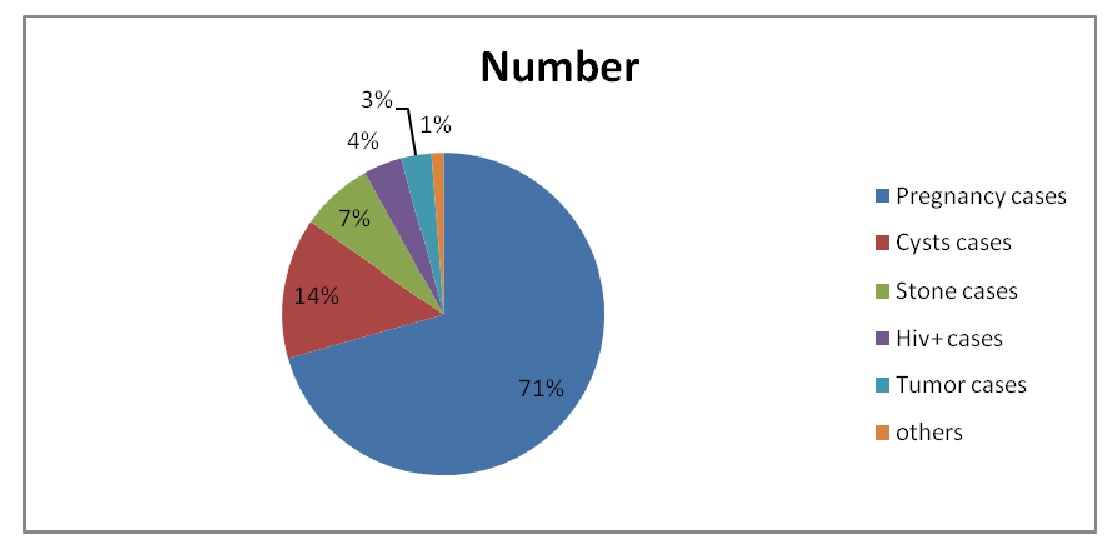

Figure 4: Pie Chart Showing the Percentage of Total Number of Patients Having Various Kinds of Health Problems.

It shows that $71 \%$ of the total patients viewed are of pregnancy cases. It shows that most of the patients coming to this ultrasound clinic are pregnant women and they are getting maximum benefits from this hospital. $14 \%$ of them are having various kinds of cyst problems. The doctor says that with the changes in lifestyles of today's modern world this health problem is also becoming very common. $7 \%$ of total patients are having stone problems, $4 \% \mathrm{HIV}+\mathrm{cases}, 3 \%$ tumor cases and $1 \%$ are others. These all kind of people requires ultrasound to get treatment and therefore ultrasound technology is beneficial for everyone.

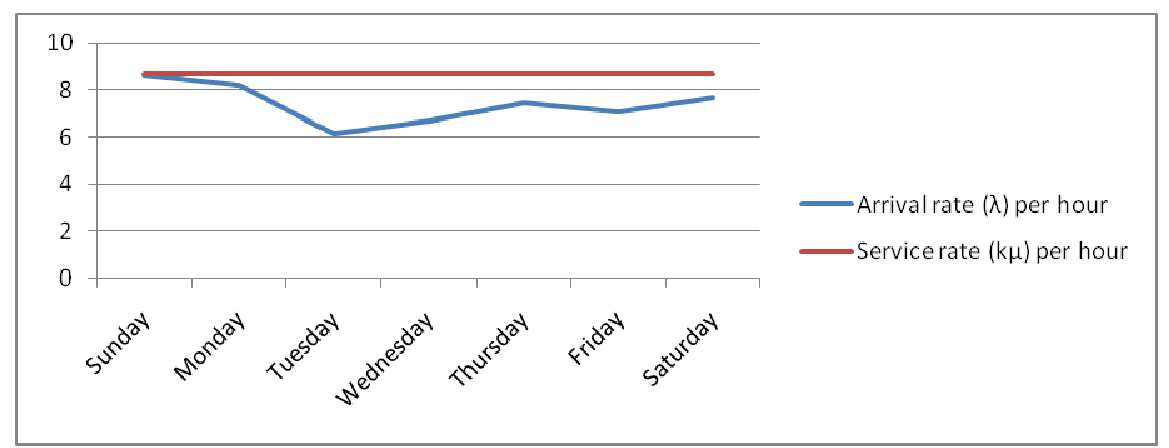

Figure 5: Patient's Arrival Rate and Service Rate on Different Weekdays.

The basic idea behind creating separate scenarios for different days of the week comes from the figure5. It was noticed that patient's arrival rate is maximum on Sunday. While arrival rate on other weekdays is less as compared to that 
of Saturday, Sunday and Monday. Increase in arrival rate on Saturday, Sunday and Monday is due to the reason that some people are free during these days. Figure6 shows the number of patients coming on each date of the month of the observation. There are a lot of variations in the arrival pattern of the patients therefore applying model to particular days of the week was a good idea to understand the things more clearly.

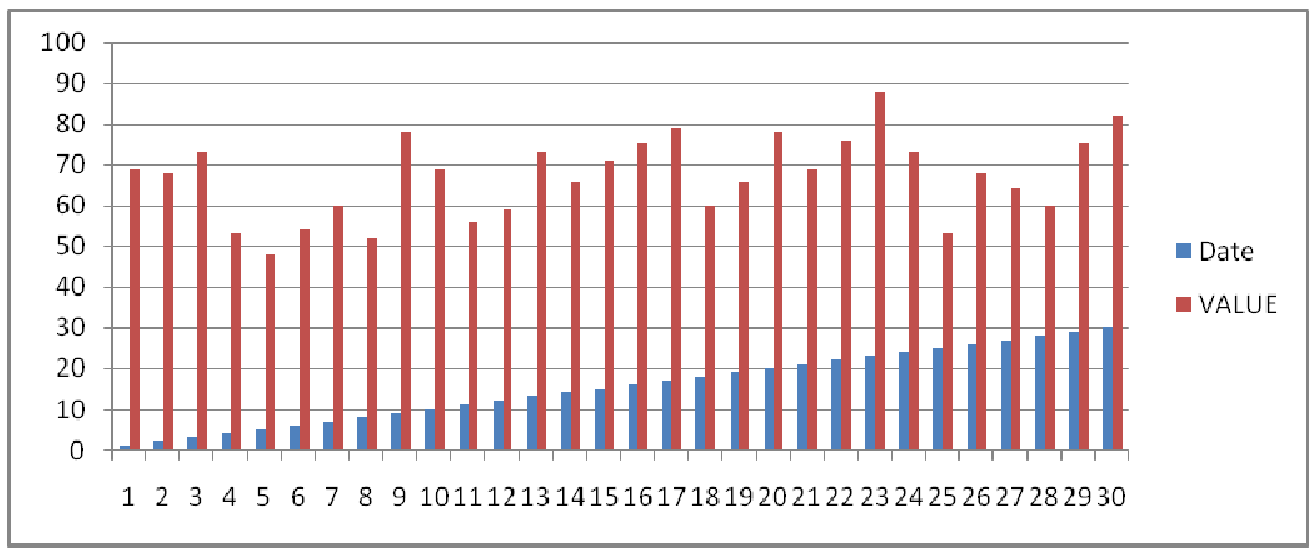

Figure 6: The Number of Patients Coming on Each date of the Month of the Observation Results and Conclusion.

Our experience in ultrasound clinic suggests that pregnant women are taking more benefit from ultrasound technology. It is clear from table1and figure5 that patient's arrival rate increases on Saturday, Sunday and Monday while the service rate remains the same during the week. To provide a service with minimum waiting, a good patient flow is required. Moreover table2 shows that maximum increase in arrival rate on Sunday cause a high waiting. Increasing number of servers in busy days of the week can act as a good way to improve the patient's flows and hence minimize waiting during these days. This study establishes that queuing theory and queuing models can act as an effective tool to study the healthcare problems and helping hospital management teams in taking various decisions regarding the number of resources required.

\section{REFERENCES}

1. Ameh, N., Sabo, B., Oyefabi, M.O.(2013) Application of queuing theory to patient satisfaction at a tertiary hospital in Nigeria. Niger Med J;54(1):64-7.

2. Haghighinejad, H. A., Kharazmi, E., Hatam, N., Yousefi, S., Hesami, S.A., Danaei, M., Askarian, M., (2016).Using Queuing Theory and Simulation Modelling to Reduce Waiting Times in An Iranian Emergency Department. IJCBNM;4(1):11-26.

3. GHIMIRE, SUSHIL, et al. "MULTI-SERVER BATCH SERVICE QUEUEING MODEL WITH VARIABLE SERVICE RATES." International Journal of Applied Mathematics and Statistical Science 6.4 (2017): 43-54.

4. Bahadori, M., Mohammadnejhad, S. M., Ravangard, R. Teymourzadeh, E. (2014). Using queuing theory and simulation model to optimize hospital Pharmacy performance. Iran Red Crescent Medical Journal; 16(3):

5. Creemers, S. and Lambrecht, M.R. (2007). Modeling a healthcare system as a queuing Network: the case of Belgian hospital. A paper submitted to department of decision sciences and information management, research centre for operations and management, Katholieke University Leuven.

6. Derlet, R. \& Richards. J. (2000). Overcrowding in the nation's emergency departments: complex causes and distributing effects, Annual of emergency Medicare, 35(1): 63-68. 
7. Hadiuzzaman, Md, et al. "Development of delay model for non-lane based traffic at signalized intersection." International Journal of Civil Engineering 3 (2014): 67-82.

8. Fomundam, Samuel F. \& Jeffrey W.Herrmann (2007): A survey of Queuing Theory Applications in Healthcare, ISR Technical Report.

9. Green, V.L., Soares, J., Giulio, J. and Green, R.(2006). Using queuing theory to increase the effectiveness of emergency department provides staffing, academic emergency medicine, 13, (1): 61-68.

10. Moharana, Shanti Swaroop, Rajadeepan D. Ramesh, and DigamberPowar. "Analysis of load balancers in cloud computing." International Journal of Computer Science and Engineering 2.2 (2013): 101-108.

11. Henry, M. C. (2001). Overcrowding in America's emergency department inpatient wards replace emergency Car, academic emergency medicine, 8 (2): $151-155$.

12. Lakshmi, C. and Iyer, S. A. (2013).Applications of Queuing theory in Healthcare : A Literature Review, pp. 25-39.

13. Mardiah, F. P. and Basri, M. H.(2013).The Analysis of Appointment System to Reduce Outpatient Waiting Time at Indonesia's Public Hospital.

14. McClain, J.O. (1976) Bed planning using queuing theory models of hospital occupancy: a sensitivity analysis. Inquiry, 13, 167-176.

15. Nosek, R.A. and Wilson, J.P. (2001) Queuing theory and customer satisfaction: a review of terminology, trends, and applications to pharmacy practice. Hospital Pharmacy, 36, 275-279

16. Soomro, Muhammad Waqas, Majid Ali Memon, and Muhammad IreanAbro. "Performance analysis of routing protocols in MANET with static and mobile nodes using HTTP traffic." International Journal of Electronics and Communication Engineering (IJECE) 1.2 (2012): 23-31.

17. Obamiro, J. K. (2012). Queuing patient flow and resource allocation in intensive care unit, Bayero business review, 4(2), 3950 (University of Bayero, Kano State).

18. Obamiro, J. K. (2013). Effects of waiting time on patient satisfaction: Nigerian hospital experience, journal of economic behaviour, 3(3): 7-17.

19. Huang,Q.,Zeng,Z.,(2017),A Review on Real-Time 3D Ultrasound Imaging Technology,HindawiBioMed Research International.

20. Shah, S.P., Epino,H., Bukhman,G., Umulisa,I., Dushimiyimana, J.M.V. Reichman,A.,Noble, V.E.(2008).Impact of the introduction of ultrasound services in a limited resource setting:rural Rwanda 2008. BMC International Health and Human Rights.

21. Afrane, S., Appah, A.(2014)Queuing theory and the management of Waiting-time in Hospitals: The case of Anglo Gold Ashanti Hospital in Ghana. International Journal of Academic Research in Business and Social Sciences. DOI: 10.6007/IJARBSS/v4i2/590 URL:http://dx.doi.org/10.6007/IJARBSS/v4-i2/590

22. Silval,J.P.Z.D., Gonçalves, W., Freitas, R.R.D.F., Gonçalves W., Menezes, G.S.D.(2019).Queue theory Applied to the care of Pregnancies related to Pregnancy, Childbirth and the Puerperium. International Journal of Advanced Engineering Research and Science (IJAERS) Vol-6, Issue-9.

23. Aung, T., Naing, L.L. (2019). Comparative Study on Different Queuing Models to Reduce Waiting Time in Brahmaso Clinic. International Journal of Scientific and Research Publications, Volume 9, ISSN 2250-3153. 
24. Obamiro, J. K. (2017). Queuing Network Analysis and Optimal Bed Determination: An Evaluation of Nigerian University Teaching Hospital Emergency Department.Proceedings of the International Conference on Industrial Engineering and Operations Management Bogota, Colombia.

25. Olorunsola, S. A, Adeleke R. A., and Ogunlade, T. O. (2014). Queueing analysis of patient flow in hospital, IOSR journal of mathematics (IOSR-JM),Volume 10, PP 47-53.

26. Vass, H. and Szabo, K. Z. (2015). Application of queuing model to patient flow in emergency department. Case studyProcedia Economics and Finance 32, $479-487$.

27. Operations Research Theory and Applications by J.K.Sharma, $4^{\text {th }}$ edition.

28. Operations Research by Prem Kumar Gupta, Dr. D.S.Hira. 